\title{
Continuum Modeling of Crowd Turbulence
}

\author{
Abhinav Golas ${ }^{1}$, Rahul Narain ${ }^{2}$, and Ming C. $\operatorname{Lin}^{1}$ \\ ${ }^{1}$ University of North Carolina at Chapel Hill \\ ${ }^{2}$ University of California, Berkeley
}

(Dated: October 28, 2014)

\begin{abstract}
With the growth in world population, the density of crowds in public places has been increasing steadily, leading to a higher incidence of crowd disasters at high densities. Recent research suggests that emergent chaotic behavior at high densities - known collectively as crowd turbulence-is to blame. Thus, a deeper understanding of crowd turbulence is needed to facilitate efforts to prevent and plan for chaotic conditions in high-density crowds. However, it has been noted that existing algorithms modeling collision avoidance cannot faithfully simulate crowd turbulence.

We hypothesize that simulation of crowd turbulence requires modeling of both collision avoidance and frictional forces arising from pedestrian interactions. Accordingly, we propose a novel model for turbulent crowd simulation, which incorporates a model for inter-personal stress and acceleration constraints similar to real-world pedestrians. Our simulated results demonstrate a close correspondence with observed metrics for crowd turbulence as measured in known crowd disasters.
\end{abstract}

PACS numbers: 89.40.-a,45.70.-n

\section{INTRODUCTION}

Crowd disasters, like those at the Hajj in Mecca and the Love Parade disaster in 2010, have shown that current crowd control methods can prove untenable at high densities. As population increases, instances of highdensity crowds can only grow more frequent. This poses a significant safety risk that can only be addressed by a deeper understanding of crowd behavior in such conditions. Recent research by Helbing et al. 1, 2] has started to shed light on the unique characteristics of crowd motion in these high-density disasters.

Under normal conditions, crowds of pedestrians typically move smoothly, with slowdowns and fluctuations only near obstacles. When the density and urgency of pedestrians increases, however, the behavior of crowds changes dramatically. First, the crowd begins to exhibit stop-and-go waves: the motion of pedestrians alternates between stopped at high density and moving forward at low density, with these density waves traveling backwards through the crowd. Second, at even higher densities, the crowd shows a chaotic, oscillatory behavior reminiscent of turbulence in fluids, with pedestrians being pushed in all directions, even backwards against their desired direction of motion. The term crowd turbulence has been introduced to describe such conditions [1].

Even under such extreme conditions, pedestrian motion shows a coherent structure. Individual motion is replaced by mass motion, and the scale of turbulent oscillations spans several times the pedestrian separation. Crowds also exhibit a stick-slip instability [1] which leads to a rupture when stress in the crowd becomes too large. As a result, the crowd splits up into clusters of different sizes, with strong velocity correlations within each cluster, and distance-dependent correlations between clusters.

Simulation can be an invaluable tool to understand the processes behind this behavior, and to formulate strate- gies to prevent their occurrence. However, existing crowd simulation techniques have been found to be inadequate for modeling crowd turbulence by [1]. We hypothesize that at high densities, physical forces between pedestrians become relevant as they are brought into close contact. Accordingly, we propose that a model for inter-personal forces is essential for simulating crowd behavior at high-densities. We use a continuum approach where inter-personal forces are represented as stress fields caused by discomfort and friction of the underlying simulated pedestrians.

Numerical simulations of real-world scenarios using our model demonstrate close qualitative and quantitative correspondence to observed data. We believe that models like ours incorporating inter-personal stress can be used to obtain a better understanding of crowd behavior in dense, panicked situations for both theoretical analysis and practical situations.

\section{BACKGROUND}

Behavior of pedestrians in public spaces has been the focus of much research over the past few decades. This includes insights obtained by observing real-world crowds [3, as well as observations made in controlled laboratory conditions [4, 5] to help improve crowd flow in public spaces. A well-known result of such research is that pedestrian speed decreases with crowd density [6-10]. This relationship is known as the fundamental diagram. Another major focus of research is to understand crowd behavior in order to help reduce the occurence of disasters [8]. Recent research has started looking into disasters at large scales and high crowd densities. Insights offered by [1] into the crowd disasters at the Hajj, and by [2] into the Love Parade disaster in particular, form the basis for our proposed model.

For large-scale or high-density crowds, simulations of- 
fer the one of very few avenues for study, as such crowds cannot be recreated safely under controlled conditions. The field of crowd simulation is quite broad and includes modeling of navigation, psychological factors, social interactions, and so on; navigation, however, is the most important factor for reproducing the dynamics of largescale crowd motion. Accordingly, in this section we review the related work in this area relevant to the problem of simulating crowd turbulence, restricting attention to models that adhere to the fundamental diagram.

Virtual crowds are traditionally modeled as collections of individual virtual agents. In this model, the navigation problem is decomposed into two sub-problems, global planning and local navigation. Global planning plots a path for each agent to its goal, while only considering static obstacles in the scene. Local navigation then attempts to guide the agent along this path while avoiding collisions with other agents in the scene. As this component is responsible for reacting to the motion of other agents, it determines how well the model can reproduce stop-and-go waves and crowd turbulence.

Local navigation algorithms can be broadly classified into discrete or continuum algorithms. Discrete algorithms [11 15] solve the navigation problem for each agent individually, considering other agents as separate entities. For efficiency reasons, only a limited set of nearby agents is considered for avoidance. The alternate approach is to consider the crowd as a continuum [16, 17, with the avoidance problem being recast as navigating around regions of congestion. This is ideally suited for medium to high densities. Narain et al. [18 propose a macro-scale collision avoidance model for calm dense crowds, though densities at which turbulence is not observed. Recently, a generic approach for long-range visibility [19] has also been proposed that allows local navigation methods to conform to the fundamental diagram without explicit modeling.

One of the key insights offered by recent studies of crowd disasters [1, 2], has been the similarity of crowds to granular materials beyond the level of analogy established in previous work [20. Therefore, we draw on ideas from granular flow simulation in developing our model. Granular materials have long been the focus of scientific study, and accurate numerical simulation of their behavior is challenging both for discrete and continuous approaches [21]. Nevertheless, several approximate continuum models have been proposed [22 24]. These models reproduce much of the qualitative properties of granular materials, and their relative computational efficiency guides our decision to use a continuum formulation for our approach.

Existing work has sought to model crowd turbulence using discrete force-based models. Yu et al. 25] propose that existing force-based models like social forces [26] can be used to recreate situations where variance of agent velocity reaches levels similar to those seen in turbulent crowds. However, their results do not demonstrate the emergent clusters of motion that is characteristic of tur- bulent flows. Similarly, the model proposed by Moussaïd et al. 12] demonstrates fluctuations in agent positions similar to turbulent crowds, but does not match any other crowd turbulence metrics like the "pressure" defined by [1, or the clustering of agents demonstrated by turbulent crowds.

We postulate that similar to granular media, an essential component in any model for turbulent crowds is inter-personal friction, as described in Section [V] This, combined with a physically-based model for agent acceleration completes our model for crowd turbulence, detailed in Section III. We have validated our model and hypothesis against two observed instances of real-world crowd turbulence, the Hajj, and the Love Parade disaster of 2010 in Section $\mathrm{V}$. We conclude with the limitations of our model and scope for future work in Section VI.

\section{CONTINUUM MODEL FOR CROWD TURBULENCE}

\section{A. Crowd as a Continuum}

Real-world crowds consist of individuals, and most exisiting crowd simulation models emulate this using discrete virtual pedestrians or agents. Doing so is essential for resolving possible pedestrian motion at low-tomedium densities. Higer density models subsume this discrete model into a continuum navigation model, where discrete agents represent pedestrians, but their motion is modeled using a continuum representation of the crowd. Since this model is primarily intended for high-density crowds, we begin with this continuum representation, similar to the Fluid Implicit Particle (FLIP) approach [27.

In the continuum formulation, the crowd state is defined in terms of two properties, density $\rho$ (people per $\mathrm{m}^{2}$ ) and current velocity $\mathbf{v}$. These properties are defined in a continuous 2-dimensional space tending to zero outside the bounds of the crowd. To couple this represention with discrete agents, we define a per-agent state using position $\mathbf{x}_{i}$ and current velocity $\mathbf{v}_{i}$ defined in two dimensions.

In particular, the density field $\rho$ can be defined as

$$
\rho(x)=\sum_{i} w\left(x-x_{i}\right),
$$

where the sum is computed over all agents, and $w$ is a smoothing kernel. In our simulations, we use the piecewise bilinear hat function with a support of $1 \mathrm{~m}$. Continuum representations of any agent property, like velocity, can then be computed as a weighted average,

$$
\mathbf{v}(x)=\frac{\sum_{i} w\left(x-x_{i}\right) \mathbf{v}_{i}}{\rho(x)} .
$$

The time evolution of the crowd in this FLIP formulation is as follows. The position of each agent changes 
according to its current velocity. However, as agents are performing active locomotion rather than being passive physical objects, their velocities must be updated through a computational procedure taking into account both their planning decisions and external forces. This is performed in two steps: first, a global planning step computes a preferred velocity $\mathbf{v}_{p}$ for each agent to direct it towards its goal. Local navigation then computes a collision-free velocity $\mathbf{v}_{c f}$ accounting for influences from other nearby agents. The collision-free velocity field is then constructed using equation (2).

In order to obtain valid behavior, these fields must be defined appropriately at boundaries, particularly near solid obstacles. Continuity of the density field, in particular, is essential to obtain correct behavior and avoid collisions with obstacles. In grid cells which are partially covered by obstacles, we compute a coverage fraction $\phi \in[0,1]$ - the fraction of the cell covered by obstacles. In such cells Equations (1) and (2) can be corrected by dividing by the fraction $(1-\phi)$. In cells completely covered by obstacles, density is defined via extrapolation. That is, the density of the cell is chosen to be that of nearest cell not completely covered by obstacles, ensuring that the gradient of density is purely tangential at obstacle boundaries. This choice allows the local collision avoidance model described in Section IIIC, and the stress model in Section IV to generate correct behavior around solid obstacles.

\section{B. Continuum Turbulence Model}

Our model for crowd turbulence consists of a local navigation algorithm that defines appropriate behavior over the full range of low to high crowd densities. Even though our primary focus is a model for crowd behavior at high densities, a model for low-medium densities is necessary to impose realistic initial and boundary conditions for high-density regions.

We assume the existence of global planner that provides a preferred velocity $\mathbf{v}_{p}$ for each agent at every time step, defining the optimal direction for the agent to reach its goal. We do not make any assumptions about its temporal continuity or smoothness, thus the preferred velocity may change sharply between time steps.

We begin by choosing an existing local navigation algorithm that satisfies the fundamental diagram. Given the preferred velocity $\mathbf{v}_{p}$, the algorithm attempts to compute a "collision-free" velocity $\mathbf{v}_{c f}$, that is, a velocity close to $\mathbf{v}_{p}$ which will not bring the agent into collision with its neighbors. Adherence to the fundamental diagram implies that as crowd density $\rho$ increases, the collision-free speed of the agent $\left|\mathbf{v}_{c f}\right|$ decreases toward zero. Agents tend towards this velocity over a characteristic time $\tau$, via an acceleration

$$
\mathbf{a}=\frac{\mathbf{v}_{c f}-\mathbf{v}_{t}}{\tau} .
$$

Such a model is appropriate for densities up to those observed in most real-world crowds, i.e. up to a density of 5.5 to 6 people per $\mathrm{m}^{2}$ [6, 9. At higher densities, collision avoidance may be impossible, as agents may be in nearconstant contact with other agents. Thus, existing collision avoidance models cannot be used directly. Therefore, we introduce a high-density model which describes the effects of these contact interactions, and whose contribution is zero below a threshold density.

In keeping with observations of [1, we postulate that crowd behavior at high densities is controlled predominantly by inter-personal stress similar to the stresses observed in granular media. Accordingly, we define the stress $\sigma$ as a symmetric $2 \times 2$ tensor field representing the internal forces in the crowd. This tensor $\sigma$ can be decomposed into its normal and deviatoric components,

$$
\sigma=p \mathbf{I}+\mathbf{s},
$$

where the normal component $p$ represents normal or repulsion forces, while the deviatoric stress $\mathbf{s}$ is trace-free and represents frictional forces. We describe how to compute $p$ and $\mathbf{s}$ in section IV] The force due to stress is

$$
\nabla \cdot \sigma=\nabla p+\nabla \cdot \mathbf{s}
$$

Therefore, the combined contribution of stress forces (4) and local navigation (3) can then be expressed by modifying the definition of agent acceleration as:

$$
\mathbf{a}=\frac{\mathbf{v}_{c f}-\mathbf{v}_{t}}{\tau}-\frac{1}{\rho}(\nabla p+\nabla \cdot \mathbf{s})
$$

The acceleration implied by this equation may be greater than what can be achieved by pedestrians in the real world. The spontaneous oscillations seen in crowd turbulence can also arise from limits on pedestrians' response to variations in the crowd, namely, their bounded accelerations. Thus, we impose a constraint that the magnitude of the actual agent acceleration must be smaller than a constant $a_{\max }$. Thus, the agent velocity $\mathbf{v}$ changes as

$$
\frac{d \mathbf{v}}{d t}=\hat{\mathbf{a}} \min \left(a_{\max },\|\mathbf{a}\|\right) .
$$

where $\hat{\mathbf{a}}$ is the direction of the acceleration vector. The agent position can then be updated through time integration.

The resulting algorithm is shown in Fig. 1.

\section{Local Collision Avoidance}

We use the collision avoidance model proposed by Golas et al. [19] for computing the collision-free velocity $\mathbf{v}_{c f}$. Our choice of this model is guided by the fact that it has been validated against real-world data for low-medium density crowds. In our model, this optimal direction is computed independently for every agent. 
- Define the crowd density field using Equation (1)

- For each agent $a$, compute optimal direction $\hat{\mathbf{v}}$ using Equation (9)

- Use Equation (2) to determine the continuum velocity field

- Compute frictional stress using this velocity field

- Advect agents and update states using Equation (7)

FIG. 1: Continuum Simulation Algorithm for Crowd Turbulence

In this model, agents move at their maximum natural speed in a direction that maximizes progress towards goal. The agent velocity can thus be expressed as $\mathbf{v}_{c f}=\hat{\mathbf{v}} f(\rho)$, where $f(\rho)$ is the average speed at the given density $\rho$. We use the same linear form for $f(\rho)$ proposed by 19 , with the exception of having the minimum value of $f(\rho)$ to be $0.01 \mathrm{~m} / \mathrm{s}$ instead of zero. Assuming that the agent maintains a constant heading along a chosen direction $\hat{\mathbf{v}}$ for a planning horizon $\Delta t$, the displacement after time $\Delta t$ will be:

$$
\mathbf{d}(\Delta t)=f \hat{\mathbf{v}} \Delta t+\frac{1}{2}(f \hat{\mathbf{v}} \cdot \nabla \rho) f^{\prime} \hat{\mathbf{v}} \Delta t^{2}
$$

where $f$ and $f^{\prime}$ are evaluated at the density at the current position. The direction $\hat{\mathbf{v}}$ is chosen to maximize progress towards goal $\mathbf{v}_{p} \cdot \mathbf{d}(\Delta t)$, giving the optimization problem

$$
\underset{\hat{\mathbf{v}}}{\arg \max }\left(\mathbf{v}_{p} \cdot \hat{\mathbf{v}}+\frac{f^{\prime} \Delta t}{2}(\hat{\mathbf{v}} \cdot \nabla \rho)\left(\mathbf{v}_{p} \cdot \hat{\mathbf{v}}\right)\right)
$$

subject to $\|\hat{\mathbf{v}}\| \leq 1$. Solving this problem using their lookahead approach yields a velocity that satisfies the fundamental diagram.

\section{INTER-PERSONAL STRESS}

We now complete our model by defining the method to compute inter-personal stress. As noted previously, under low-to-medium density conditions, local collision avoidance computes a path that avoids collisions with other agents. At higher densities, agents may be unable to avoid collisions. In such constrained conditions, we postulate that agent behavior is governed predominantly by the desire to avoid or minimize the likelihood of contact and collisions with other agents and static elements in the scene. This implies that when possible, agents attempt to maximize inter-agent separation, as that would reduce the likelihood of collisions. At a macroscopic scale, this is equivalent to the crowd as a whole trying to attain a state with lower mean density. This is analogous to the role of pressure in fluids and granular materials.

Considering the crowd as a compressible fluid, we define a pressure-like field $p$ as a monotonically increasing function of density $\rho$. Its effect is to allow density fluctuations to propagate through the crowd, similar to the waves observed in turbulent crowds. In order to avoid confusion with the "pressure" metric [1] used later in the paper, we call this functional form discomfort. The behavior described previously can thus be expressed as a desire by the crowd to lower discomfort caused by high densities and cramped spaces by moving along its gradient. By ensuring that the gradient of density is tangential to obstacles in Section III A, we ensure that pressure gradient is tangential to obstacles as well, ensuring that the component of agent velocity normal to obstacles is zero.

To define a functional form for discomfort, we make certain observations:

1. Discomfort must be a constant below a critical density of 3.5 people per $\mathrm{m}^{2}$, i.e. its gradient is zero. Pedestrians at such densities can move freely towards their goal, and thus we expect virtual agents to do the same.

2. At higher densities, discomfort rises steeply. This is expected as pedestrians still have available space to navigate and reach lower density regions.

3. Beyond a certain yield density of 5.5 to 6 people per $\mathrm{m}^{2}$ the gradient of discomfort reduces and plateaus. This is a reflection of the fact that at such densities, pedestrians would lose the available space to navigate and thus have a more limited set of responses.

We note that a number of functions can follow these considerations and yield similar results. We choose a form similar to one proposed by 28, where a similar concept of discomfort is proposed:

$$
g(\rho)= \begin{cases}0, & \text { if } \rho<\rho_{\text {crit }} \\ c_{1} \frac{\rho}{\sqrt{\rho_{\text {yield }}-\rho+\epsilon}}-c_{2}, & \rho_{\text {crit }} \leq \rho<\rho_{\text {yield }} \\ c_{3} \rho-c_{2}, & \rho \geq \rho_{\text {yield }}\end{cases}
$$

where $\epsilon$ is a small positive constant, $c_{1}$ is a parameter, and $c_{2}$ and $c_{3}$ are chosen to ensure continuity of the function at $\rho_{\text {crit }}$ and $\rho_{\text {yield }}$. The function grows steeply to the yield point $\rho_{\text {yield }}$ after which it grows linearly. Specific values used for these constants as used in our simulations are defined in Section V.

The other essential component of inter-personal stress is friction. Without modeling friction, stick-slip instabilities observed in turbulent crowds cannot be obtained. Friction modeled here represents the combination of two possible responses. The first is the friction between agents in contact, which in turbulent crowds can cause physical damage and even rip clothes off of people [1. The second follows from the original intent of pedestrians to avoid collisions. At very high densities, relative motion between agents is likely to cause contact. In such situations, maintaining the relative configuration amongst 
agent neighbors can be a viable strategy to avoid collisions. At a macroscopic scale, this corresponds to clusters of agents moving coherently.

Though the use of friction as part of a collision avoidance algorithm has been suggested in different contexts, these approaches have modeled only one kind of friction [29. In order to obtain stick-slip instabilities, it is essential to model both static and kinetic friction, along with an appropriate yield condition that controls the transition between the two. Static friction prevents relative motion between two bodies in contact, while kinetic friction acts to oppose relative velocity between two bodies already having relative motion. A number of models exist in the domain of granular materials to model such phenomena. We choose a computationally efficient continuum model 30, which we find is sufficient to replicate stick-slip instabilities.

Based on the maximum dissipation principle, this formulation computes the frictional stress which minimizes the kinetic energy of the crowd flow, as defined using velocity from Equation (7). However, the frictional stress is constrained by a yield criterion, which relates the magnitude of frictional stress to the amount of normal stress, which in our case is discomfort. In keeping with [30, we use the Drucker-Prager yield criterion:

$$
\|\mathbf{s}\|_{F} \leq \sqrt{3} \alpha g(\rho)
$$

where $\|\mathbf{s}\|_{F}=\sqrt{\sum s_{i j}^{2}}$ is the Frobenius norm, and $\alpha$ is the coefficient of friction.

The kinetic energy after a time step $\Delta t$ can be approximated [30] as

$$
E=\frac{1}{2 \rho_{\text {yield }}} \int\left\|\rho\left(\mathbf{v}_{t}-\nabla p\right)+\Delta t \nabla \cdot \mathbf{s}\right\|^{2} d V,
$$

which is a quadratic functional of the frictional stress field $\mathbf{s}$. The value of $\mathbf{s}$ is determined by minimizing $E$ subject to the yield criterion (11). Upon discretizing $\mathbf{s}$ on the simulation grid and approximating the yield criterion by an intersection of half-spaces, we obtain a constrained quadratic programming problem, which can be solved using standard methods. We obtain efficient convergence using the solver proposed by [31].

\section{VALIDATION}

We ran simulations using our model to recreate the behavior observed in two crowd disasters. For all simulations, the critical density $\rho_{\text {crit }}$ was 3.5 people per $\mathrm{m}^{2}$, yield density $\rho_{\text {yield }}=5.5$ people per $\mathrm{m}^{2}$, constants $c_{1}=13.33, c_{2}=1.5$, with $\epsilon=0.01$. We use a friction coefficient $\alpha=0$ at densities less than $\rho_{\text {crit }}, \alpha=0.75$ at densities greater than $\rho_{\text {yield }}$, with linearly interpolated values for $\rho \in\left[\rho_{\text {crit }}, \rho_{\text {yield }}\right]$. We use a second-order midpoint method for time integration.

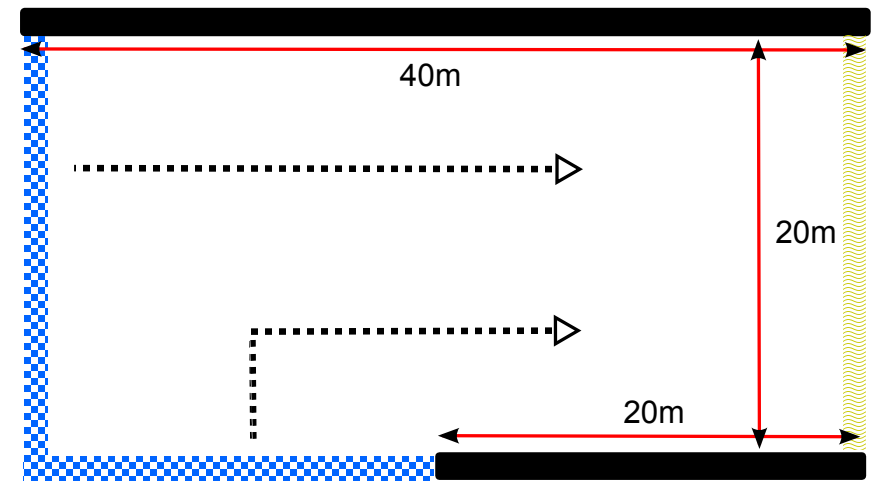

FIG. 2: (Color online) Scene setup for Hajj simulations.

The scene consists of two merging sets of agents emerging from the inlets shown in the blue checkerboard pattern, following the path highlighted by dotted arrows and exiting via the wavy yellow outlet on the right. Corridor dimensions are shown by red arrows.

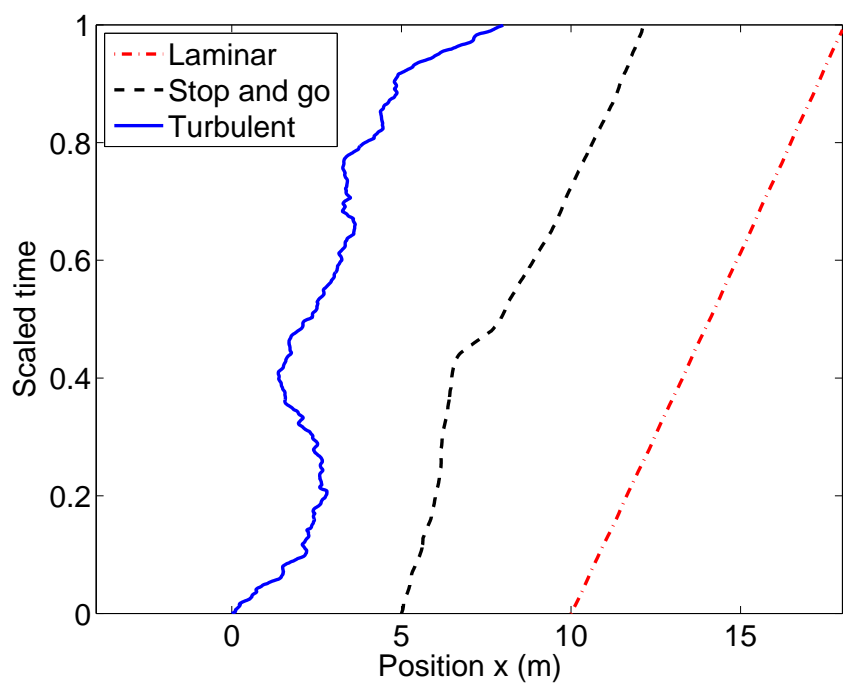

FIG. 3: (Color online) Representative trajectories taken by agents in the Hajj simulation to travel a distance of $8 \mathrm{~m}$ in laminar (red, right), stop-and-go (black, middle) and turbulent flow (blue, left), with time rescaled to unity.

Animated visualizations of the simulations generated by our crowd turbulence model in the following two scenarios can be found online菲

\footnotetext{
1 http://gamma.cs.unc.edu/CrowdTurbulence

2 See Supplementary Material at [] for animated visualizations of simulations
} 


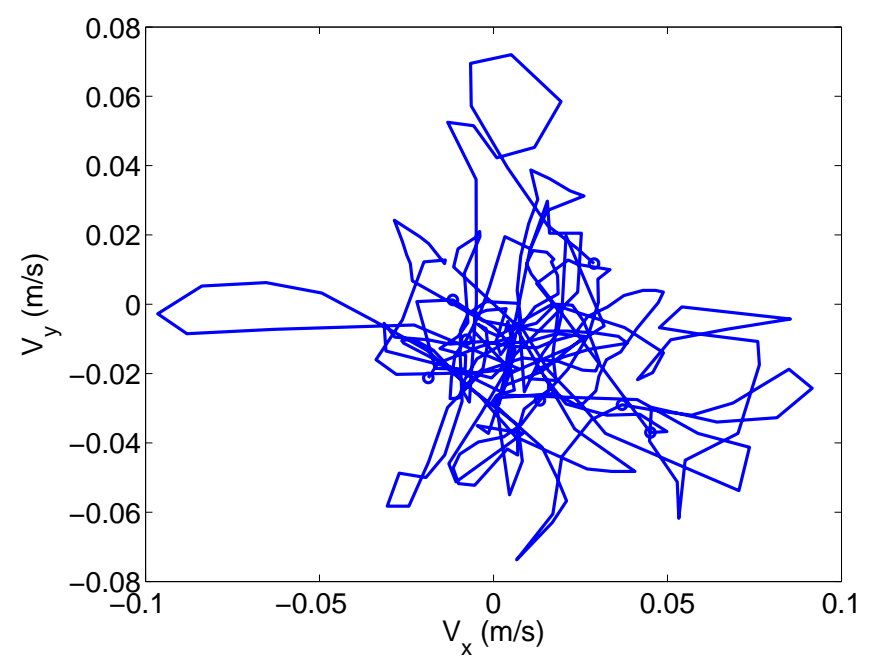

FIG. 4: (Color online) Temporal evolution of velocity components $v_{x}$ and $v_{y}$. Under laminar flow $v_{y}$ will remain close to zero. However, under turbulent flow, such as in this case, we observe motion that is orthogonal and even against the desired direction of motion (along the $+x$ direction).

\section{A. Hajj}

The Hajj is an Islamic pilgrimage to the city of Mecca, where millions of people undertake every year. Over the past two decades, it has been the location of multiple crowd disasters resulting in the loss of over a thousand lives. As a result, it has also been the focus of research in crowd analysis and simulation. Simulations have been performed on a representative scene proposed by [2, 25]. The scene setup is shown in Fig. 2 .

We ran simulations for a wide range of average densities, from 3.5 people per $\mathrm{m}^{2}$ (4400 agents) to 6 people per $\mathrm{m}^{2}$ (6800 agents). We observe the emergence of stop-andgo waves even at densities at lower end of our range, while at the higher end of the range we observe the emergence of crowd turbulence. As seen in Fig. 5a, the variation of speed vs. density follows a similar decreasing trend as observed for other models and real-world data. At densities below 5 people per $\mathrm{m}^{2}$, behavior is primarily dictated by the existing local navigation model [19]. Since their model utilizes a linear representation of the fundamental diagram, slope of the curve at these densities follows a linear trend. At densities higher than 5 people per $\mathrm{m}^{2}$, our model exhibits a close correspondence with the speed trend noted by [1], as well as similar magnitudes.

We use the "pressure" metric $P$ defined by Helbing et al. 1] as an indicator of crowd turbulence:

$$
P(\mathbf{x}, t)=\rho(\mathbf{x}, t) \operatorname{Var}_{\mathbf{x}, t}(\mathbf{v})
$$

where $\operatorname{Var}_{\mathbf{x}, t}(\mathbf{v})$ is the variance of velocity. In practice, we only compute this variance with respect to either space or time. Previous work shows that real crowds become

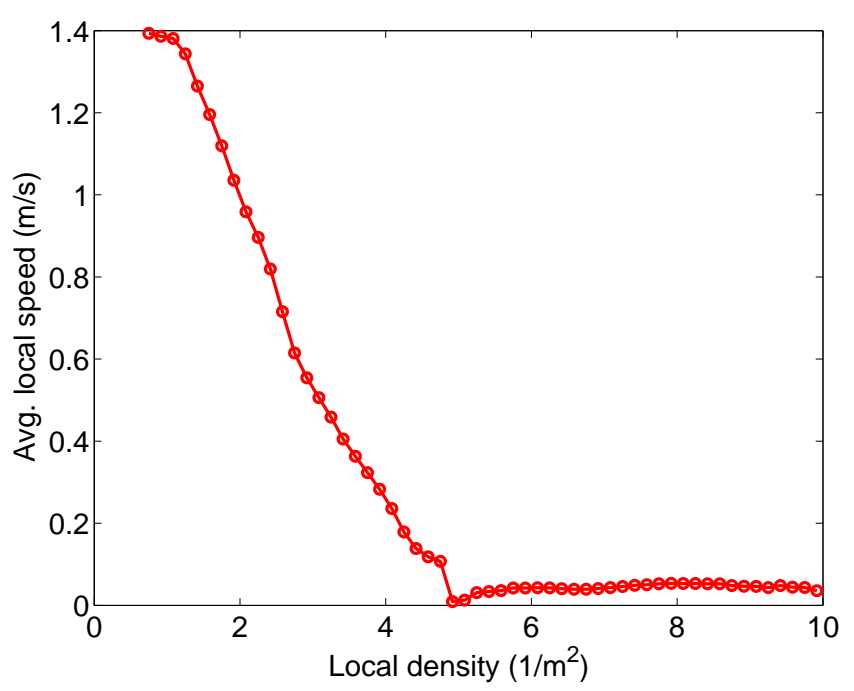

(a)

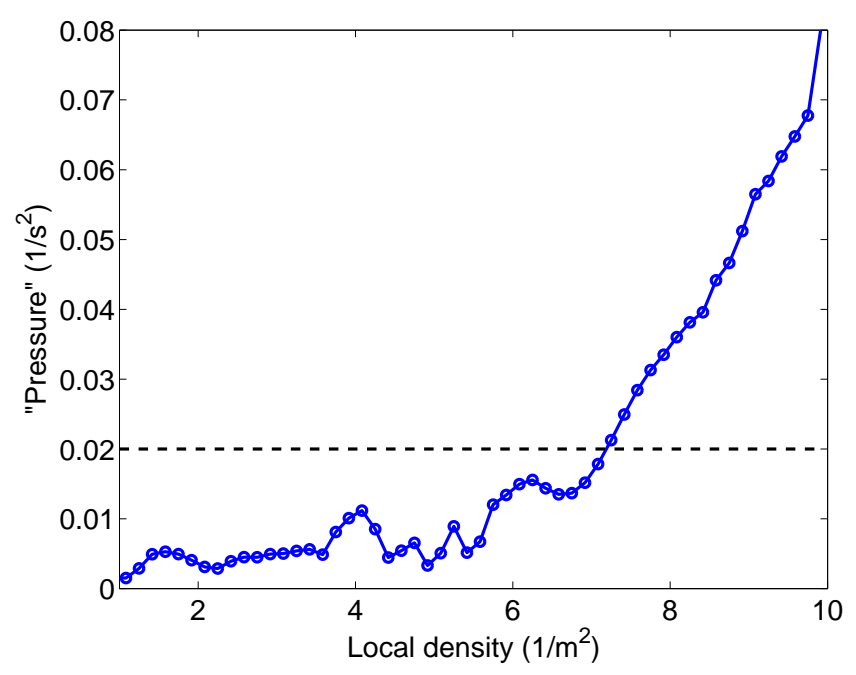

(b)

FIG. 5: (Color online) (a) Plot of average speed vs. density. Note that even though average speeds may be low at high densities, high variance implies that agent speeds may be significantly higher than the mean

(b) Plot of crowd "pressure" $P(\mathbf{x})=\rho(\mathbf{x}) \operatorname{Var}_{\mathbf{x}}(\mathbf{v})$ as defined by [1]. We observe values greater than 0.02 at local densities of 7 people per $\mathrm{m}^{2}$ and higher, which are indicative of crowd turbulence.

turbulent at values of $P$ greater than 0.02 . As shown in Fig. 5b, our simulation replicates turbulent crowd conditions at densities greater than 7 people per $\mathrm{m}^{2}$.

Fig. 3 shows representative trajectories for agents moving in the Hajj scene. Under laminar flow conditions, agents move unhindered along their desired direction of motion, forward along the $x$-axis. At the onset of stopand-go flow, we observe that agents sometimes come to a standstill, as denoted by the trajectory becoming vertical for a short period of time. However, at the onset of turbulent flow, we see that agents can even be pushed 


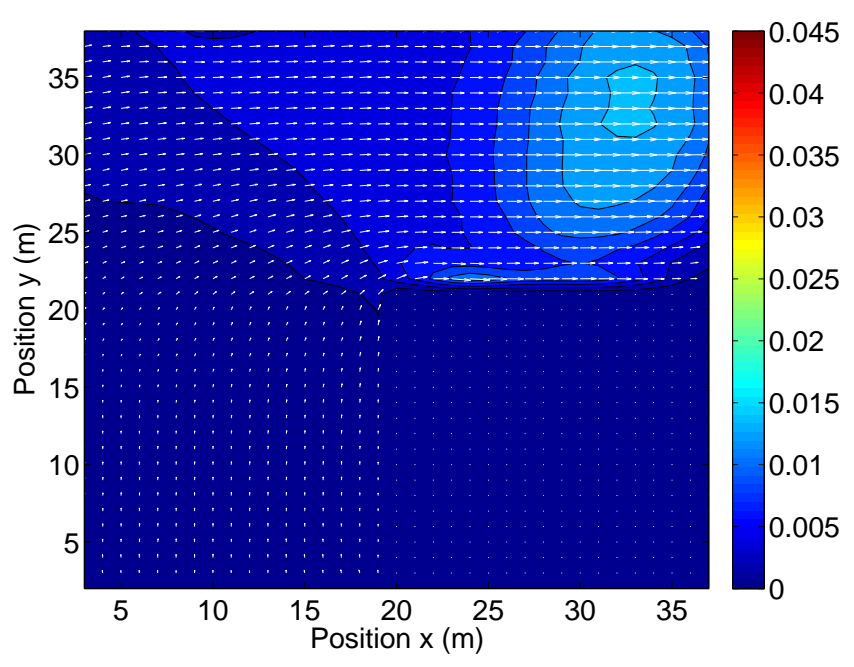

(a)

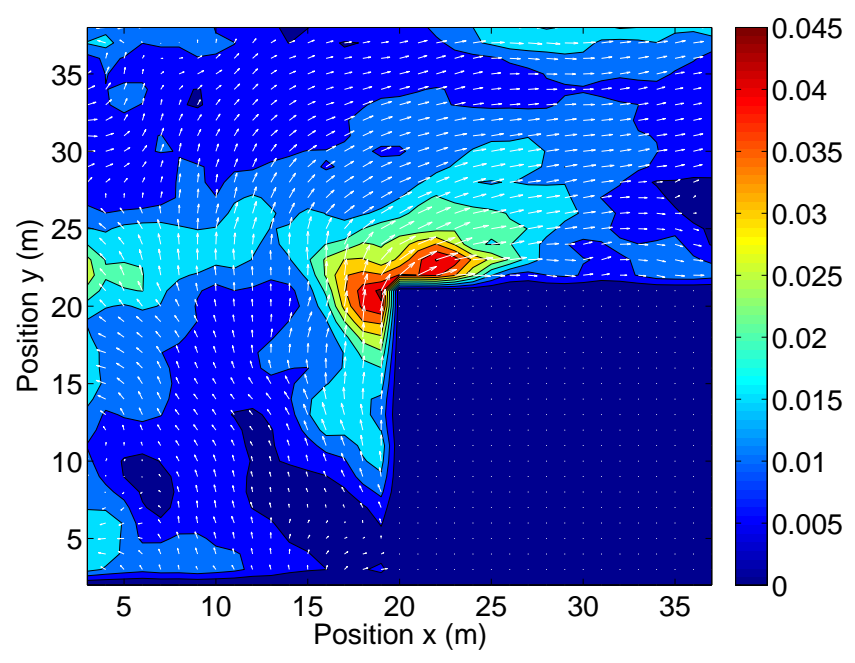

(b)

FIG. 6: (Color online) Plot of crowd "pressure" overlaid with mean flow velocities for a time period of 3 seconds for (a) stop and go flow, and (b) turbulent flow. Note formation of irregular clusters as denoted by isocontour lines. Color bars show range of "pressures" observed; note that values greater than 0.02 do not arise in the occurrence of stop-and-go waves.

backwards against their will. Fig. 4 shows that in such cases, agents may move in nearly any direction, including directions orthogonal and opposite to their intended direction of motion, as shown by the variation of the velocity components $v_{x}$ and $v_{y}$.

Fig. 6a and Fig. 6b demonstrate the cohesive flow that arises at the onset of stop-and-go waves and turbulence respectively. At the onset of stop-and-go waves, a concentration of agents develops behind and in front of the rarefaction wave. As these disturbances intensify into crowd turbulence, we see creation of agent clusters as illustrated by irregular isocontours in Fig. 6b. The stick-

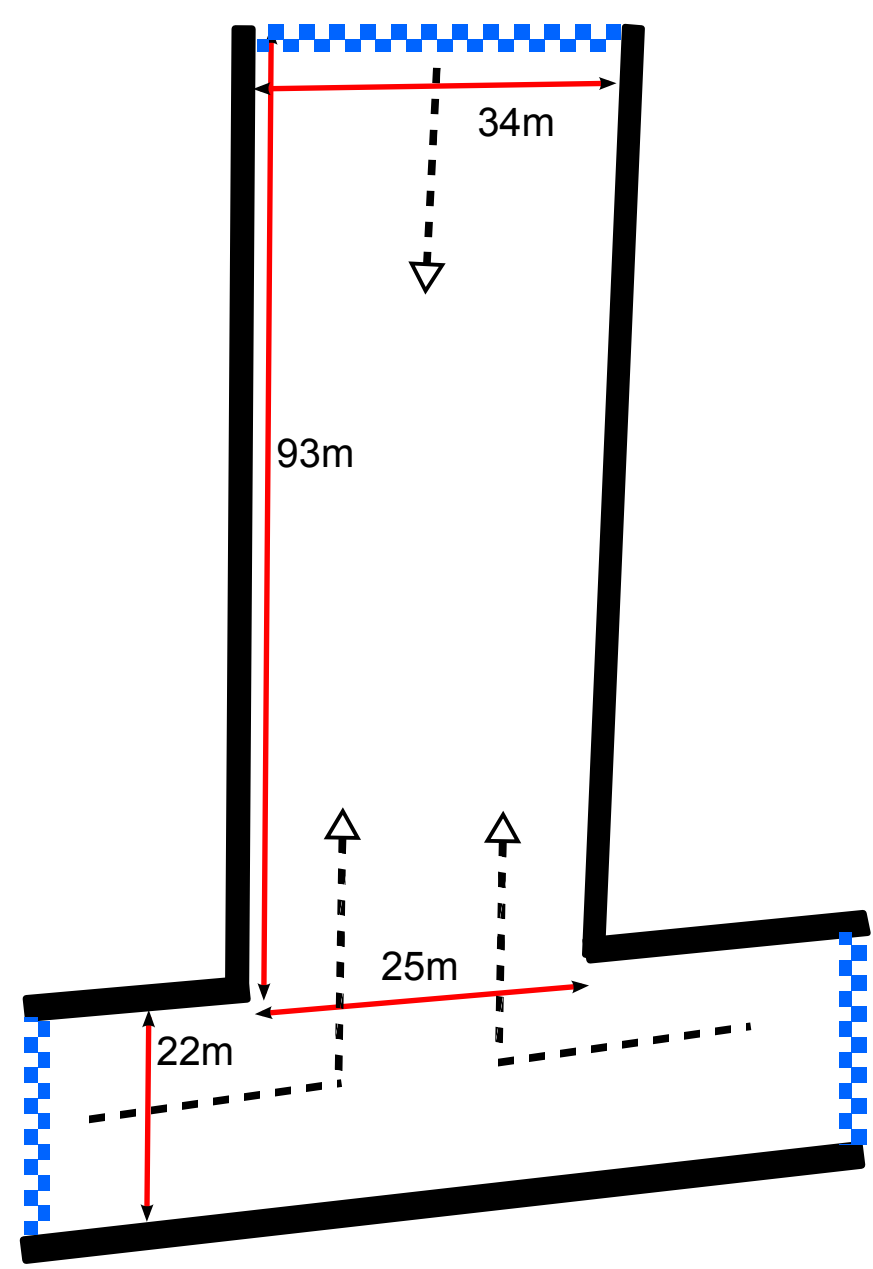

FIG. 7: (Color online) Scene setup for Love Parade simulations. The scene consists of three merging sets of agents, emerging from the inlets shown in the blue checkerboard pattern. Agents from the bottom two inlets proceed towards the third inlet at the top and vice versa, following the paths highlighted by the dotted arrows. Corridor dimensions are shown by red arrows.

slip instability manifests at the boundaries of these clusters. As demonstrated by these plots, our model is able to replicate known observations of crowd turbulence at the Hajj.

\section{B. Love Parade 2010}

The Love Parade was an annual electronic dance music festival held in Germany. At the event in 2010, held at a railway yard in Duisburg, Germany, a crowd control failure led to the 21 casualties from suffocation, with more than 510 people injured. Though the venue was intended for 250,000 people, up to 500,000 people reportedly attended, with about 3200 police present for crowd control. Due to miscommunication between the various police groups, two inlets were opened allowing a large 


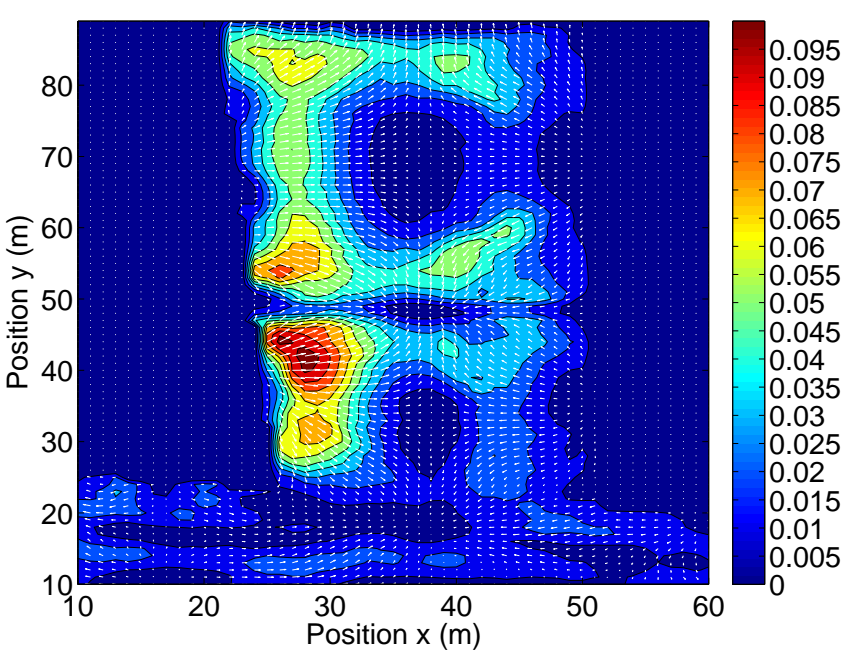

FIG. 8: (Color online) Plot of crowd "pressure" overlaid with mean flow velocities for a time period of 3 seconds for turbulent flow. Note formation of irregular clusters as denoted by isocontour lines. Color bars show range of "pressures" observed.

influx of pedestrians to enter a corridor blocked by an outflux of pedestrians wanting to leave the concert. The resulting bottlenecked situation caused turbulent conditions to develop, leading to multiple casualties.

Based on known data from the venue, we set up a representative scene to model a portion of the railway yard where the mishap occurred. This scene setup is shown in Fig. 7. Recently, the disaster was analyzed in [2] and an official documentary ${ }^{3}$ reinforced the view that crowd turbulence was responsible for the casualties.

We note that in our simulations, we observe turbulence at densities of 5.5 people per $\mathrm{m}^{2}$ and higher, as seen in Fig. 9b, and observe turbulent oscillations similar to those seen in the official documentary $(5: 42-5: 52)$. As seen in Fig. 8 most intense turbulence is observed in the region where incoming and outgoing agents meet. In addition, we see formulation of clusters with stick-slip instabilities similar to those observed in the Hajj scenario as demonstrated by variation in "pressure" throughout the crowd.

\section{Importance of Friction in Simulating Crowd Turbulence}

In order to validate the hypothesis that inter-personal friction is essential for simulating crowd turbulence, we simulated the Hajj scenario with discomfort alone. As is evident from the "pressure" plot in Fig. 10, without the added contribution of friction, turbulence is not observed.

3 "Official Documentary of the Love Parade 2010 Disaster", http: //www . youtube . com/watch?v=8y73-71FBNE (2010).

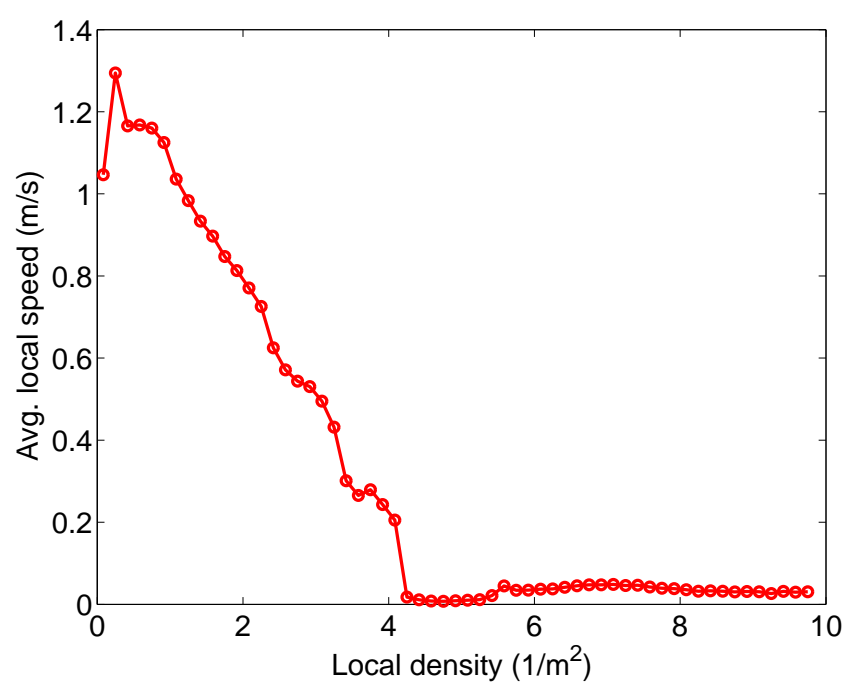

(a)

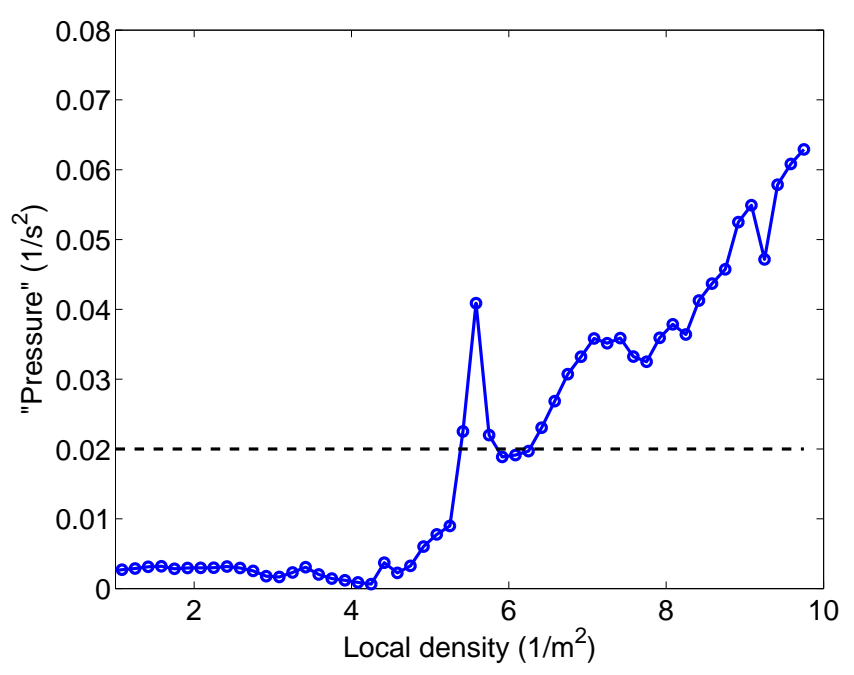

(b)

FIG. 9: (Color online) (a) Plot of average speed v.s. density. (b) Plot of crowd "pressure". We observe values greater than 0.02 at local densities of 5.5 people per $\mathrm{m}^{2}$ and higher, which are indicative of crowd turbulence.

As noted earlier, cluster formation is one of the hallmarks of crowd turbulence. A plot of the magnitude of the strain rate tensor $\left\|\nabla v+(\nabla v)^{T}\right\|_{F}$ in Fig. 11 shows isocontours delineating variation in velocity. These variations indicate shear regions where agents slip against each other, thereby marking cluster boundaries. Such a transition cannot occur without friction, underlining its importance in a crowd turbulence model.

\section{CONCLUSION}

In this paper, we have postulated that modeling interpersonal forces is necessary for simulating crowd turbu- 


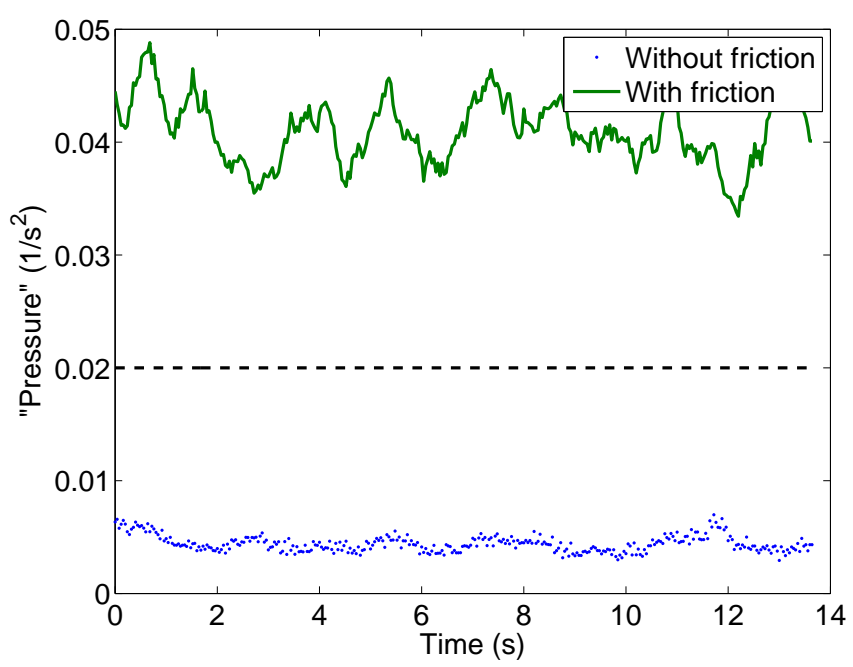

FIG. 10: (Color online) Plot of crowd "pressure" $P(t)=\left\langle\rho(t) \operatorname{Var}_{t}(\mathbf{v})\right\rangle_{\mathbf{x}}$ in the Hajj scenario with both discomfort and friction (top), and with discomfort alone

(bottom). $\langle\cdot\rangle_{\mathbf{x}}$ denotes the mean computed over the entire scene. Note how discomfort alone is unable to recreate turbulent flow conditions.

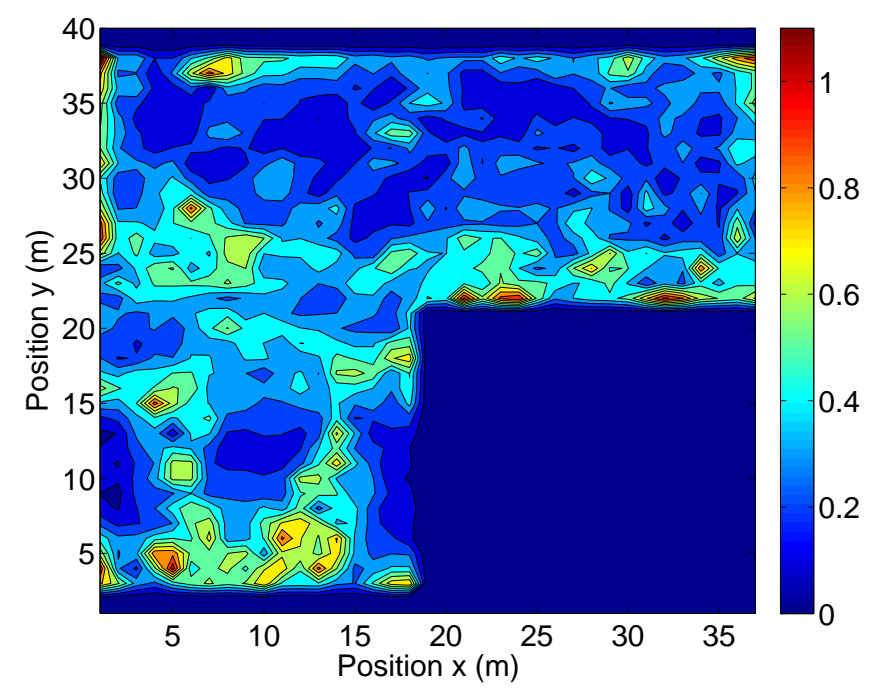

FIG. 11: (Color online) Plot of the magnitude of the strain rate tensor $\left\|\nabla v+(\nabla v)^{T}\right\|_{F}$ at $t=3 s$ for turbulence flow in Hajj scenario. Note cluster formation as shown by isocontours. lence. To validate this hypothesis we propose a novel model for turbulent crowds, using which we are able to simulate stop-and-go waves as well as chaotic behavior symptomatic of crowd turbulence. These reproduce the features of well-known crowd disasters at the Hajj and the Love Parade disaster of 2010. Our model also shows good correspondence with quantitative metrics proposed for detecting crowd turbulence and establishes the importance of modeling friction for simulating crowd turbulence.

Our model can simulate such crowds with globally computed stress at interactive rates on current generation PCs. We believe this finding opens up possibilities of real-time response to crowd conditions that may pose threats of developing into crowd turbulence. It should also prove to be a valuable aid for safety planning and disaster prevention, by allowing planners to test designs for the possibility of crowd turbulence.

The numerical validation shown in this paper has focused on the limited set of metrics reported in previous work. Our model is consistent with real-world data as measured by these metrics. The lack of detailed realworld datasets prevents more extensive validation of the model, our hypothesis, and the underlying forces.

In order to ensure efficient simulation, we compute stress using the solver used by [30] which approximates the Frobenius norm using the infinity norm. Though the error of such an approximation is bounded, it may cause minor deviations from expected behavior. A solver that can determine stress under Frobenius norm constraints could improve accuracy of computed results.

Though our model closely matches data from real instances of crowd turbulence, we believe that results can be further improved with automatic parameter tuning [35] and validation of our model with more real-world data. Expanding the limited set of publicly available data from crowd disasters with fine-grained information about pedestrian trajectories and velocities would allow the development of significantly more accurate models.

\section{ACKNOWLEDGEMENTS}

Thanks to Sean Curtis for discussions and other help with this research. This research was supported in part by the Army Research Office, National Science Foundation, and UNC Arts and Sciences Foundation.
[1] D. Helbing, A. Johansson, and H. Z. Al-Abideen, "Dynamics of crowd disasters: An empirical study," Phys. Rev. E 75, 046109 (2007)

[2] D. Helbing and P. Mukerji, "Crowd disasters as systemic failures: analysis of the Love Parade disaster," EPJ Data Science 1, 7 (2012)
[3] J.J. Fruin, Pedestrian planning and design (Metropolitan Association of Urban Designers and Environmental Planners, 1971).

[4] J. Zhang, W. Klingsch, A. Schadschneider, and A. Seyfried, "Transitions in pedestrian fundamental diagrams of straight corridors and T-junctions," J. Stat. 
Mech. 2011, P06004 (2011).

[5] J. Zhang, W. Klingsch, A. Schadschneider, and A. Seyfried, "Ordering in bidirectional pedestrian flows and its influence on the fundamental diagram," Journal of Statistical Mechanics: Theory and Experiment 2, 2 (2012), arXiv:1107.5246 [physics.soc-ph].

[6] L. A. Pipes, "An operational analysis of traffic dynamics," J. Appl. Phys. 24, 274 - 281 (1953).

[7] A. Polus, J. Schofer, and A. Ushpiz, "Pedestrian flow and level of service," Journal of Transportation Engineering 109, 46-56 (1983)

[8] J. Fruin, "The causes and prevention of crowd disasters," in Engineering for Crowd Safety, edited by R. A. Smith and J. F. Dickie (Elsevier, 1993).

[9] U. Weidmann, Transporttechnik der Fussgaenger, 2nd ed., 90 (1993).

[10] A. Seyfried, B. Steffen, W. Klingsch, and M. Boltes, "The fundamental diagram of pedestrian movement revisited," J. Stat. Mech. (2005).

[11] S. J. Guy, J. Chhugani, S. Curtis, P. Dubey, M. Lin, and D. Manocha, "PLEdestrians: a least-effort approach to crowd simulation," in Proceedings of the 2010 ACM SIGGRAPH/Eurographics Symposium on Computer Animation, SCA '10 (2010).

[12] M. Moussaïd, D. Helbing, and G. Theraulaz, "How simple rules determine pedestrian behavior and crowd disasters," Proceedings of the National Academy of Sciences (2011), 10.1073/pnas.1016507108

[13] A. Portz and A. Seyfried, "Modeling stop-and-go waves in pedestrian dynamics," ArXiv e-prints (2010), arXiv:1001.3283 [physics.soc-ph]

[14] S. Curtis and D. Manocha, "Pedestrian simulation using geometric reasoning in velocity space," Pedestrian and Evacuation Dynamics (2012).

[15] C. W. Reynolds, "Flocks, herds and schools: A distributed behavioral model," ACM SIGGRAPH 21, 25-34 (1987).

[16] R. L. Hughes, "The flow of human crowds," Annual Review of Fluid Mechanics 35, 169-182 (2003)

[17] A. Treuille, S. Cooper, and Z. Popovic, "Continuum crowds," ACM Trans. Graph. 25, 1160-1168 (2006).

[18] R. Narain, A. Golas, S. Curtis, and M. C. Lin, "Aggregate dynamics for dense crowd simulation," ACM Trans. Graph. (2009).

[19] A. Golas, R. Narain, S. Curtis, and M. C. Lin, "Hybrid long-range collision avoidance for crowd simulation," IEEE Transactions on Visualization and Computer Graphics 20, 1022-1034 (2014, Preliminary version appeared in the Proc. of ACM I3DG 2013)
[20] S.P. Hoogendoorn, S. Luding, P.H.L. Bovy, M. Schreckenberg, and D.E. Wolf, Traffic and Granular Flow '03 (Springer, 2007).

[21] R. Behringer, H. Jaeger, and S. Nagel, "Introduction to the focus issue on granular materials," Chaos: An Interdisciplinary Journal of Nonlinear Science 9, 509-510 (1999)

[22] M. Quecedo, M. Pastor, M. I. Herreros, and J. A. Fernández Merodo, "Numerical modelling of the propagation of fast landslides using the finite element method," International Journal for Numerical Methods in Engineering 59, 755-794 (2004).

[23] Igor S. Aranson and Lev S. Tsimring, "Continuum description of avalanches in granular media," Phys. Rev. E 64, 020301 (2001).

[24] C. Josserand, P.-Y. Lagrée, and D. Lhuillier, "Stationary shear flows of dense granular materials: a tentative continuum modelling," The European Physical Journal E: Soft Matter and Biological Physics 14, 127-135 (2004).

[25] W. Yu and A. Johansson, "Modeling crowd turbulence by many-particle simulations," Phys. Rev. E 76, 046105 (2007)

[26] D. Helbing and P. Molnár, "Social force model for pedestrian dynamics," Physical Review E 51, 4282 (1995).

[27] J. U. Brackbill and H. M. Ruppel, "FLIP - A method for adaptively zoned, particle-in-cell calculations of fluid flows in two dimensions," Journal of Computational Physics 65, 314-343 (1986).

[28] R. L. Hughes, "A continuum theory for the flow of pedestrians," Transportation Research Part B: Methodological 36, $507-535$ (2002)

[29] D. Helbing, I. Farkas, and T. Vicsek, "Simulating dynamical features of escape panic," Nature (2000).

[30] R. Narain, A. Golas, and M. C. Lin, "Free-flowing granular materials with two-way solid coupling," in $A C M$ SIGGRAPH Asia 2010 Papers, SIGGRAPH ASIA '10 (ACM, New York, NY, USA, 2010) pp. 173:1-173:10.

[31] Zdeněk D. and Joachim S., "Minimizing quadratic functions subject to bound constraints with the rate of convergence and finite termination," Comput. Optim. Appl. 30, 23-43 (2005).

[32] http://gamma.cs.unc.edu/CrowdTurbulence

[33] See Supplementary Material at |] for animated visualizations of simulations.

[34] "Official Documentary of the Love Parade 2010 Disaster", http://www . youtube.com/watch?v=8y73-71FBNE (2010).

[35] S. J. Guy, J. van den Berg, W. Liu, R. Lau, M. C. Lin, and D. Manocha, "A statistical similarity measure for aggregate crowd dynamics," ACM Trans. Graph. 31, 190:1190:11 (2012). 